\title{
Analyses of body weight patterns in growing pigs: a new view on body weight in pigs for frequent monitoring
}

\author{
A. H. Stygar ${ }^{1 \dagger}$, K. A. Dolecheck ${ }^{2}$ and A. R. Kristensen ${ }^{1}$ \\ ${ }^{1}$ Department of Veterinary and Animal Sciences, University of Copenhagen, Grønnegårdsvej 2, DK-1870 Frederiksberg C, Denmark; ${ }^{2}$ Department of Animal and Food \\ Sciences, University of Kentucky, Lexington, KY 40546, USA
}

(Received 29 June 2016; Accepted 24 May 2017; First published online 24 July 2017)

Frequent BW monitoring of growing pigs can be useful for identifying production (e.g. feeding), health and welfare problems. However, in order to construct a tool which will properly recognize abnormalities in pigs' growth a precise description of the growth process should be used. In this study we proposed a new model of pig growth accounting for daily fluctuations in BW. Body weight measurements of 1710 pigs (865 gilts and 843 barrows) originating from five consecutive batches from a Danish commercial farm were collected. Pigs were inserted into a large pen (maximum capacity $=400$ ) between November 2014 and September 2015. On average, each pig was observed for 42 days and weighed 3.6 times a day when passing from the resting to feeding area. Altogether, 243,160 BW measurements were recorded. A multilevel model of pig growth was constructed and fitted to available data. The BW of pigs was modeled as a quadratic function of time. A diurnal pattern was incorporated into the model by a cosine wave with known length $(24 \mathrm{~h})$. The model included pig effect which was defined as a random autoregressive process with exponential correlation. Variance of within-pigs error was assumed to increase with time. Because only five batches were observed, it was not possible to obtain the random effect for batch. However, in order to account for the batch effect the model included interactions between batch and fixed parameters: intercept, time, square value of time and cosine wave. The gender effect was not significant and was removed from the final model. For all batches, morning and afternoon peaks in the frequency of visits to the feeding area could be distinguished. According to results, pigs were lighter in the morning and heavier in the evening (minimum BW was reached around $1000 \mathrm{~h}$ and maximum around $2200 \mathrm{~h}$ ). However, the exact time of obtaining maximum and minimum BW during the day differed between batches. Pigs had access to natural light and, therefore, existing differences could be explained by varying daylight level during observations periods. Because the diurnal amplitude for pig growth varied between batches from 0.9 to $1.4 \mathrm{~kg}, B W$ monitoring tools based on frequent measurements should account for diurnal variation in BW of pigs. This proposed description of growth will be built into a monitoring tool (a dynamic linear model) and applied to farm data in future studies.

Keywords: automatic growth monitoring, BW, diurnal pattern, pigs

\section{Implications}

Body weight is a frequently reported trait in biological studies. For example, we want to know how BW changes with age, during a disease or after applying a therapy. However, so far we have been neglecting the fact that BW can change also during a day. We showed that BW of pigs during 1 day on average fluctuates from 0.9 to even $1.4 \mathrm{~kg}$. These fluctuations are highly statistically significant and should be included in mathematical models describing growth. The knowledge on BW rhythm might be useful in designing experiments, interpreting results or developing monitoring tools.

\footnotetext{
${ }^{\dagger}$ E-mail: as@sund.ku.dk
}

\section{Introduction}

It has been assumed that the growth of pigs is well described. For pigs observed during short time intervals, growth has been defined simply by linear functions with the intercept representing initial BW and the slope expressing an increase in BW (Toft et al., 2005). For pigs observed over longer time intervals, periods with faster and slower growth rates have been expressed in the form of Gompertz functions (Niemi et al., 2015). Such description of growth is adequate for traditional BW monitoring, which involves, for example, moving pigs from pens to a weighing crate on a daily, weekly or monthly bases. More frequent BW monitoring can be useful for production monitoring as well as identifying health 
and welfare problems (Cornou and Kristensen, 2013). However, for automatic growth monitoring to be useful, a more precise description of growth might be necessary.

In particular, diurnal growth patterns may be necessary to account for when multiple BW measurements are taken within 1 day. Mäntysaari and Mäntysaari (2015) suggested that, in dairy cattle, BW varies throughout the day according to udder, gut and bladder fill. Indeed, they found that twicedaily BW measurements from 230 dairy cattle were an average $7.3 \mathrm{~kg}$ lower in the morning than in the afternoon. Because both measurements were taken after milking, a minimal effect of udder fill was assumed. Instead, the diurnal influence was attributed to the cows eating more during the daytime than during the nighttime. Similarly, multiple recent studies using automatically collected data have identified diurnal feeding patterns in pigs (Fernández et al., 2011; Hoy et al., 2012; Andretta et al., 2016). Although it is intuitive to think BW of pigs might also follow a diurnal pattern, no previous research appears to have verified that assumption.

This study is part of larger project, aimed at designing a tool able to alert the farmer about deterioration in pigs' growth. In order to alert the farmer of unexpected changes in growth, precise description of growth on an hourly basis has to be known.

The main objective of this study was to describe the relationship between the BW of pigs and time expressed in hours and to evaluate the assumption that the BW of pigs follows a diurnal cyclic pattern. In the presented study, the number of registered visits to the feeding area was also investigated. Frequencies of visits to the feeding area were compared with the obtained diurnal amplitude of pigs' growth.

\section{Material and methods}

\section{Data from the herd}

The PiglT project is a strategic research alliance founded to improve welfare and productivity in growing pigs using advanced information and communication technologies methods (Kristensen and Jensen, 2015). The BW data used in this study was collected on a commercial herd of finishing pigs within the PiglT project. The herd included a finisher unit with four large common pens, each with a maximum capacity of 400 pigs. Each large common pen measured $14.0 \mathrm{~m}$ by $9.0 \mathrm{~m}$ and included a main area for resting, two feeding areas with 18 feeding points each, and a slaughter collection area.

In this herd, the main area of each pen was separated from the feeding area and the slaughter collection area by $a$ weight sorting system (Agrisys-Nedap, Groenlo, The Netherlands, graphically illustrated on Figure 1). Each time a pig wanted to enter the feeding area it had to pass through the weighing system. When a pig entered the weighing system, the scale doors closed, the pig was identified using an individual electronic ear tag, weight was recorded and then the system directed the pig to whichever feeding area it was assigned (based on weight). After feeding, pigs could exit the feeding area and enter the main area via a one-way door. Alternatively, once the pig reached slaughter weight it was

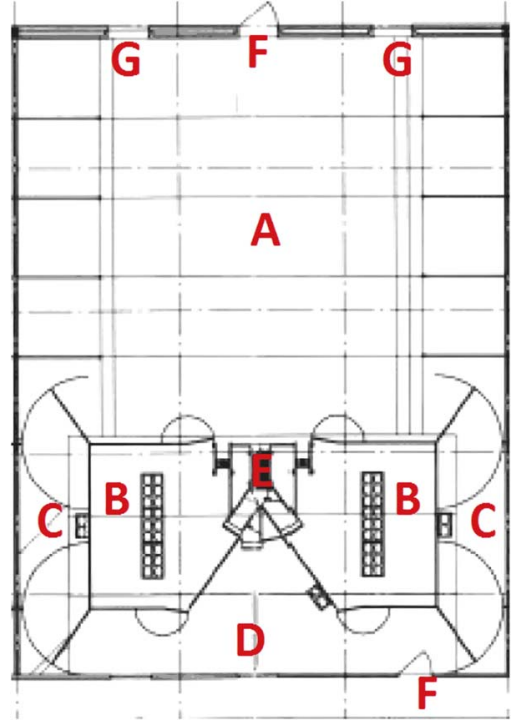

Figure 1 Overview of the large common pen finisher unit that data was collected from for a study on daily changes in pig BW where (A) main area, (B) feeding area, (C) relief area, (D) sorting area, (E) weighing scale with 24-h lamp, (F) doors and (G) windows (from Krogsdahl 2015).

automatically directed into the slaughter collection area. Any sick pigs, when identified by the herd caretaker, were moved to a section for sick animals.

In the studied farm all of the pigs originated from a crossbred dam (Landrace $\times$ Yorkshire) and a pure bred sire (Duroc), which is a common combination in Denmark. Pigs entered the large common pen finisher unit in mixed-sex groups when they reached $\sim 30 \mathrm{~kg}$. The sex of weighed pigs was identified using electronic ear tags (gilts were marked with even numbers while barrows were marked with odd numbers). The pigs were allowed a 5-week adaptation period to learn the weight sorting system. During that time, all doors between the main area and feeding area were open. Following the adaption period, weight was recorded each time each pig entered the feeding area until slaughter. In the case that two pigs entered the scale simultaneously, BW was not registered. For each batch, BW data were registered from the time of turning on scales until the last pigs were sent to slaughter (example of all BW recordings for Batch 1 is presented in Figure 2). All pigs were fed ad libitum. For a detailed description of the feeding strategy, see Krogsdahl (2015).

In this study, BW measurements of pigs from Pen 1 (Batch 1 , Batch 3, Batch 4, Batch 5) and Pen 2 (Batch 2) were obtained and analysed. Batches were observed during different seasons. Batch 1 and 2 were observed during winter, Batch 3 during spring, Batch 4 during summer and Batch 5 in autumn. A summary of all BW data used in this study is presented in Table 1. Altogether, 243,160 BW measurements were collected from 1710 pigs. All collected records were used in the analyses.

Pigs were exposed to both natural light (placing of doors and windows marked in Figure 1) and artificial light (24-h lamp over weighing scale). The farm had a combi-diffuse ventilation system. Temperature in the production unit was 
Table 1 Summary of data from five batches used in a study of daily pig growth

\begin{tabular}{|c|c|c|c|c|c|c|c|c|}
\hline \multirow[b]{2}{*}{ Batch } & \multirow{2}{*}{$\begin{array}{l}\text { Insertion } \\
\text { date }\end{array}$} & \multirow{2}{*}{$\begin{array}{c}\text { No. of } \\
\text { observation days }{ }^{1}\end{array}$} & \multicolumn{2}{|c|}{ No. of } & \multirow{2}{*}{$\begin{array}{c}\text { Total no. of } \\
\text { BW measurements }\end{array}$} & \multirow{2}{*}{$\begin{array}{c}\text { Average no. of } \\
\text { observation (per pig/day) }\end{array}$} & \multirow{2}{*}{$\begin{array}{c}\text { Initial mean } \\
\text { BW of pigs }(\mathrm{kg})^{2}\end{array}$} & \multirow{2}{*}{$\begin{array}{c}\text { Initial SD in } \\
\text { BW of pigs }(\mathrm{kg})^{2}\end{array}$} \\
\hline & & & Gilts & Barrows & & & & \\
\hline 1 & 25.11 .2014 & 60 & 166 & 164 & 54,322 & 3.55 & 57.5 & 9.9 \\
\hline 2 & 08.12 .2014 & 64 & 165 & 158 & 51,237 & 3.66 & 62.1 & 8.3 \\
\hline 3 & 02.03 .2015 & 58 & 178 & 173 & 43,130 & 3.61 & 61.6 & 9.9 \\
\hline 4 & 08.06 .2015 & 58 & 177 & 178 & 51,700 & 3.38 & 59.9 & 8.0 \\
\hline 5 & 15.09 .2015 & $35^{3}$ & 179 & 170 & 42,771 & 3.77 & 55.0 & 9.7 \\
\hline
\end{tabular}

${ }^{1}$ Number of days from turning on the scales until the last recorded delivery of pigs from given pen to the slaughterhouse.

${ }^{2}$ Based on the first recorded observation.

${ }^{3}$ The smaller number of observations was due to an error in saving data in the database.

regulated by the sprinklers that were installed above the main, feeding and collecting area and by the climate computer. Further descriptions of animals, labor and management can be found in Krogsdahl (2015).

\section{Time corrections}

In order to properly account for the diurnal pattern in all batches, the time of BW measurements was corrected for daylight saving time (DST). The DST indicator was obtained using the approach of Grolemund and Wickham (2011), implemented in R. Time of each BW observation was checked for DST changes. If BW was observed during central European summer time, the time of observation was reduced by $1 \mathrm{~h}$.

Due to the training period (when pigs were passing through scales without having their BW registered) the first initial BW information was obtained at 29 days after insertion in the pen. Therefore, for this analysis, the initial observation time $\left(t_{0}=0\right)$ was set at $696 \mathrm{~h}$ (29 days) after insertion.

\section{Statistical analysis}

Methods similar to Stygar and Kristensen (2016) were used to describe the BW of pigs in the herd. The fixed part of the model presented by Stygar and Kristensen (2016) was supplemented with fixed effects for the amplitude and frequency of a cosine wave (used to account for the diurnal pattern in daily BW). Due to the small number of observed batches (five consecutive batches were followed), it was not possible to estimate the random slope and intercept for the batch effect in the final model. Instead, the fit using generalized least squares method was applied to obtain a representation of the BW of a particular pig at a given hour. However, in order to account for the variation between batches, the final model was supplemented with interactions between batches and the fixed elements of the model. In summary, the full model used in the analyses of BW for pig $i$ in batch $j$ over time $t$ was described as:

$$
\begin{aligned}
y_{i j t}= & \left(\beta_{0}+q_{0 j}\right)+\left(\beta_{1}+q_{1 j}\right) t+q_{2 j} t^{2}+\left(\beta_{2}+q_{3 j}\right) \cos (w t) \\
& +\left(\beta_{3}+q_{4 j}\right) \sin (w t)+A_{i j t}+\epsilon_{i j t} \\
& A_{i j t} \sim N\left(0, \sigma_{A t}^{2}\right), \epsilon_{i j t} \sim N\left(0, \sigma_{t}^{2}\right)
\end{aligned}
$$

in which $\beta_{0}, \beta_{1}$ are the fixed herd effects of intercept and time while $\beta_{2}$ and $\beta_{3}$ are fixed effects for the cosine wave, $q_{0 j}, q_{1 j}, q_{2 j}, q_{3 j}, q_{4 j}$ are the fixed effects of intercept, time, square value of time and cosine wave assumed independent for different batches, $A_{i j t}$ is a random effect of pig and $\epsilon_{i j t}$ is a random residual. Parameters $\beta_{2}, \beta_{3}, q_{3 j}$ and $q_{4 j}$ determine the amplitude and $w$ is the length of the cosine wave used to account for the diurnal pattern in daily BW. Here, $w$ is constant, does not vary with pig or batch, and was calculated as $w=2 \pi / 24$ (representing exactly 1 day).

In addition, a gender coefficient was tested. The $P$-value for the gender coefficient suggested no significant differences between growth of gilts and barrows $(P=0.52)$, and was therefore removed from the final model.

The model assumed that the within-batch errors are allowed to be heteroscedastic. The following models specifying within-group variance were tested: fixed variance, different variances per stratum, power of covariate, exponential of covariate and constant plus power of covariate. The model which successfully described the within-group variance was constant plus power of covariate. This variance model was given by the equation:

$$
\sigma_{A t}^{2}+\sigma_{t}^{2}=\sigma_{A}^{2}\left(\delta_{1}+|t|^{\delta_{2}}\right)^{2}+\sigma^{2}\left(\delta_{1}+|t|^{\delta_{2}}\right)^{2}
$$

in which $\sigma_{A}^{2}$ is the variance in animal effect, $\sigma^{2}$ is the variance in measurement error, $\delta_{1}$ and $\delta_{2}$ are constants for the variance model estimated from data.

Since the BW data consisted of repeated measurements on the same pigs, the pig effect was assumed to be a first order autoregressive process with mean 0 and autocorrelation $\rho$ so that

$$
A_{i j t}=\rho\left(\sigma_{A t} / \sigma_{A, t-1}\right) A_{i j, t-1}+\omega_{i j t}
$$

in which $\omega_{i j t} \sim N\left(0,\left(1-\rho^{2}\right) \sigma_{A t}^{2}\right)$.

The observations of $B W$ for pigs were not equally spaced in time. Therefore, similar to Pinheiro and Bates (2000) we used continuous-time within-group correlation models, which naturally accommodate the imbalance in the data. Tested correlation models included: linear, rational quadratic, spherical, Gaussian and exponential. Similar to Stygar and Kristensen (2016), the most adequate within-group correlation model for pig BW data was found when assuming that the correlation between BW measurements of a single pig decreases exponentially with time. 
Following the approach of Pinheiro and Bates (2000) the significance of terms used in the fixed effect model were tested by the conditional $F$-tests in the single-argument form of the ANOVA for fitted models. The ANOVA method was also used to test the significance of the heteroscedastic model. Spatial correlation models were compared based on information criteria statistics (Akaike information criterion and Bayesian information criterion).

For data analyses and plotting, the R statistical software ( $R$ Core Team, 2014) and nlme package (Pinheiro et al., 2017) was used.

\section{Results}

To get an overview of the collected data, BW measurements from the five batches were plotted and examined. On average, each pig was observed for 42 days and had 3.6 registered weights per day. At both the daily and hourly level, increasing variance between pigs over time was evident (Figure 2) as previously identified by Stygar and Kristensen (2016). In addition, a diurnal pattern in hourly BW measurements was identified as seen in Figure 3.

\section{Growth model with diurnal pattern}

All parameters describing the BW of pigs from the studied herd (together with 95\% confidence intervals and $P$-values

Body weight of pigs inserted 2014-11-25

$\begin{array}{llllllllllllll}30 & 40 & 50 & 60 & 70 & 80 & 90 & 30 & 40 & 50 & 60 & 70 & 80 & 90\end{array}$

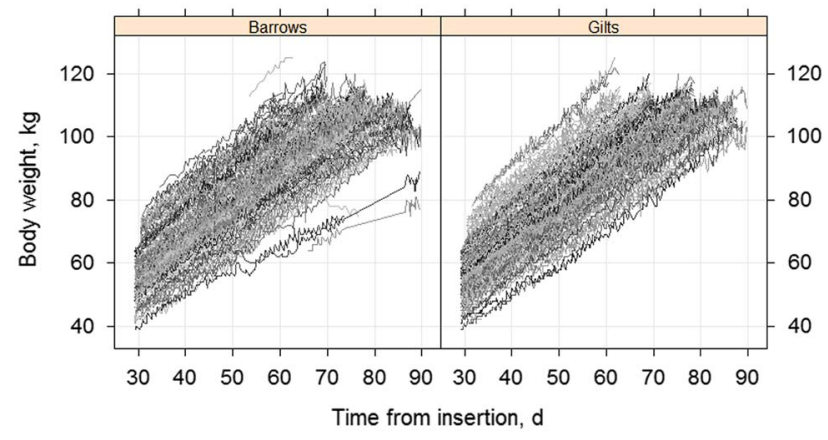

Figure 2 Body weight of all pigs from Batch 1. Each line represents the BW of an individual pig.

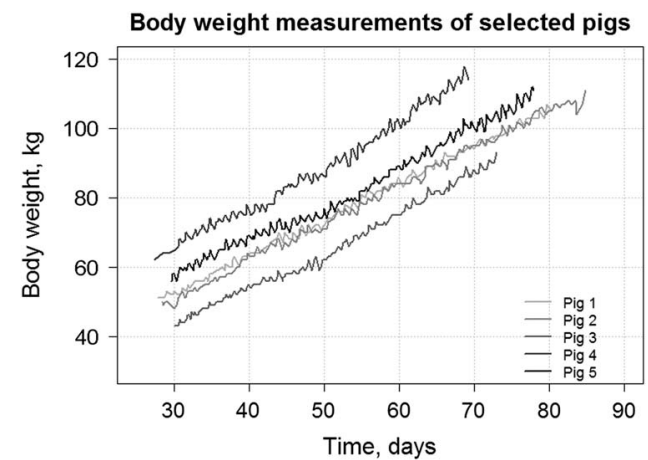

Figure 3 Body weight measurements collected during the whole fattening period of selected pigs from pen number 1 . used for testing the fixed effects of the model), obtained from the model fit, are summarized in Table 2.

According to the results, the lowest initial BW of pigs was identified in Batch $2(51 \mathrm{~kg})$ while the highest was identified in Batch $3(60.5 \mathrm{~kg})$. The herd daily gain was $\sim 1.2 \mathrm{~kg}$. The highest daily gain was obtained for Batch 2 (around $1.3 \mathrm{~kg} /$ day) whereas the lowest was obtained for Batch 5 (around $1.0 \mathrm{~kg} / \mathrm{day}$ ). Pigs were lighter in the morning (the minimum of the frequency wave was obtained around $1000 \mathrm{~h}$ ) and heavier in the evening (the maximum of the frequency wave was obtained around $2200 \mathrm{~h}$ ) (Figure 4). However, the exact time of obtaining maximum and minimum BW during the day differed between batches. In this herd, daily variation in BW of pigs was $\sim 1.2 \mathrm{~kg}$. The lowest daily amplitude in BW of pigs was observed for Batch $5(0.9 \mathrm{~kg})$ whereas the highest daily amplitude in BW was found for Batch $4(1.4 \mathrm{~kg})$.

\section{Frequency of visits to the feeding area}

In all of the batches, morning and afternoon peaks in the frequency of visits could be distinguished. However, for Batch 3 and Batch 4 the morning feeding peak was earlier in the day than the other observed batches (Figure 5). For example, $4.9 \%$ of all registrations for Batch 3 were taken between 0600 and $0659 \mathrm{~h}$. For the same batch between 0900 and $0959 \mathrm{~h}$ the frequency of observations was $5.0 \%$. Contrary, for Batch 1 the frequency of observations between 0600 and $0659 \mathrm{~h}$ was only $2.3 \%$, while between 0900 and $0959 \mathrm{~h}$ the frequency increased to $5.3 \%$.

\section{Discussion}

\section{Diurnal pattern of pig growth}

In this study, the BW of pigs was modeled as a quadratic function of time. A diurnal pattern was incorporated into the model by a cosine wave with known length $(24 \mathrm{~h})$.

In 1938, Baldwin and Kendeigh (1938) confirmed the existence of daily rhythms in the BW of multiple wild species of birds. They demonstrated that BW variation of the smaller passerine species amount to as much as $12 \%$ of the mean daily BW. However, this varied with differences in air temperature, amount of feeding and other activities. Their study identified that all birds lost BW at night and gained BW the following day. The loss of BW during the night was explained by lower air temperature whereas a gradual weight increase during the day was due to active feeding. Although the study on daily variation in BW of wild birds continued (see for example the review by Clark (1979) or Macleod et al. (2005)), not much attention has been given to daily variation in $\mathrm{BW}$ of farm animals. Daily measurements of farm animals are obviously far easier to obtain than those of wild living species. However, especially for pigs, until recent times it was not easy to collect frequent information on individual BW without the need of handling animals, which was both time consuming and expensive. One might suspect that for animals that have constant access to feed and are kept in more or less constant temperatures the daily variation in BW will 
Table 2 Parameters used to describe diurnal and periodic changes to BW of pigs in the examined herd during the fattening period $^{1}$

\begin{tabular}{|c|c|c|c|c|}
\hline Parameter $^{2}$ & Symbol & Value & $95 \%$ confidence interval & $P$-value \\
\hline Herd fixed effect for intercept & $\beta_{0}$ & 56.38 & \pm 0.78 & $<0.01$ \\
\hline Herd fixed effect for time & $\beta_{1}$ & $4.90 \times 10^{-2}$ & $\pm 1.24 \times 10^{-3}$ & $<0.01$ \\
\hline Herd fixed effect for the cos wave & $\beta_{2}$ & $57.70 \times 10^{-2}$ & $\pm 1.43 \times 10^{-2}$ & $<0.01$ \\
\hline Herd fixed effect for the sin wave & $\beta_{3}$ & $-25.92 \times 10^{-2}$ & $\pm 1.44 \times 10^{-2}$ & $<0.01$ \\
\hline Intercept $\times$ Batch 2 & $q_{02}$ & -5.22 & \pm 1.18 & $<0.01$ \\
\hline Intercept $\times$ Batch 3 & $q_{03}$ & 4.13 & \pm 1.09 & $<0.01$ \\
\hline Intercept $\times$ Batch 4 & $q_{04}$ & 1.94 & \pm 1.09 & $<0.01$ \\
\hline Intercept $\times$ Batch 5 & $q_{05}$ & -2.53 & \pm 1.09 & $<0.01$ \\
\hline Time $\times$ Batch 2 & $q_{12}$ & $0.49 \times 10^{-2}$ & $\pm 2.14 \times 10^{-3}$ & $<0.01$ \\
\hline Time $\times$ Batch 3 & $q_{13}$ & $-0.26 \times 10^{-2}$ & $\pm 1.78 \times 10^{-3}$ & $<0.01$ \\
\hline Time $\times$ Batch 4 & $q_{14}$ & $-0.36 \times 10^{-2}$ & $\pm 1.76 \times 10^{-3}$ & $<0.01$ \\
\hline Time $\times$ Batch 5 & $q_{15}$ & $-0.92 \times 10^{-2}$ & $\pm 1.88 \times 10^{-3}$ & $<0.01$ \\
\hline Quadratic time $\times$ Batch 1 & $q_{21}$ & $-1.22 \times 10^{-6}$ & $\pm 9.68 \times 10^{-7}$ & 0.01 \\
\hline Quadratic time $\times$ Batch 2 & $q_{22}$ & $-5.43 \times 10^{-6}$ & $\pm 1.16 \times 10^{-6}$ & $<0.01$ \\
\hline Quadratic time $\times$ Batch 3 & $q_{23}$ & $-6.88 \times 10^{-6}$ & $\pm 1.13 \times 10^{-6}$ & 0.2 \\
\hline Quadratic time $\times$ Batch 4 & $q_{24}$ & $-9.29 \times 10^{-8}$ & $\pm 1.01 \times 10^{-6}$ & 0.8 \\
\hline Quadratic time $\times$ Batch 5 & $q_{25}$ & $5.66 \times 10^{-6}$ & $\pm 1.51 \times 10^{-6}$ & $<0.01$ \\
\hline Cos wave $\times$ Batch 2 & $q_{32}$ & -0.03 & $\pm 2.18 \times 10^{-2}$ & $<0.01$ \\
\hline Cos wave $\times$ Batch 3 & $q_{33}$ & -0.36 & $\pm 1.16 \times 10^{-2}$ & $<0.01$ \\
\hline Cos wave $\times$ Batch 4 & $q_{34}$ & -0.01 & $\pm 2.06 \times 10^{-2}$ & 0.3 \\
\hline Cos wave $\times$ Batch 5 & $q_{35}$ & -0.15 & $\pm 2.05 \times 10^{-2}$ & $<0.01$ \\
\hline Sin wave $\times$ Batch 2 & $q_{42}$ & -0.02 & $\pm 2.18 \times 10^{-2}$ & 0.06 \\
\hline Sin wave $\times$ Batch 3 & $q_{43}$ & -0.30 & $\pm 2.05 \times 10^{-2}$ & $<0.01$ \\
\hline Sin wave $\times$ Batch 4 & $q_{44}$ & -0.15 & $\pm 2.03 \times 10^{-2}$ & $<0.01$ \\
\hline Sin wave $\times$ Batch 5 & $q_{45}$ & 0.10 & $\pm 2.10 \times 10^{-2}$ & $<0.01$ \\
\hline Evolution error & $\sigma_{A 0}^{2}$ & $4.60 \times 10^{-5}$ & $\pm 2.29 \times 10^{-5}$ & \\
\hline Observational error & $\sigma_{0}^{2}$ & $5.53 \times 10^{-7}$ & $\pm 3.61 \times 10^{-8}$ & \\
\hline Correlation $^{3}$ & $\rho$ & $99.57 \times 10^{-2}$ & $\pm 2.76 \times 10^{-4}$ & \\
\hline Power of variance model & $\delta_{1}$ & 0.96 & \pm 0.03 & \\
\hline Constant of variance model & $\delta_{2}$ & 1056.1 & \pm 242.29 & \\
\hline
\end{tabular}

${ }^{1}$ BW for pig $i$ in batch $j$ over time $t$ was described using equation (1).

${ }^{2}$ Estimates of intercept $\times$ Batch 1, time $\times$ Batch 1, Cos wave $\times$ Batch 1 and Sin wave $\times$ Batch 1 were equal to 0 .

${ }^{3}$ Assuming $24 \mathrm{~h}$ elapsed between measurements.

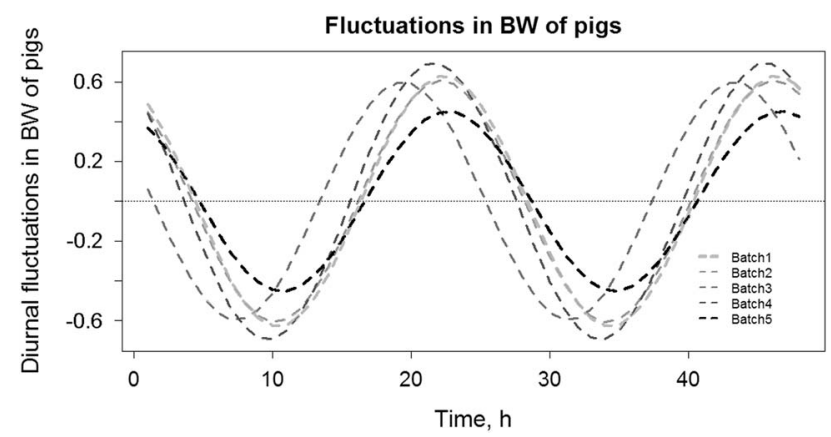

Figure 4 Diurnal fluctuations in the BW of pigs from each batch estimated from the developed model.

be small. However, recently, Mäntysaari and Mäntysaari (2015) demonstrated clear diurnal changes in BW of Nordic Red cows which had feed available $24 \mathrm{~h}$. In their study, the average difference between morning and evening BW of the cow was $7.3 \mathrm{~kg}$. The authors are not aware of studies that have evaluated the daily variation in BW of pigs.
Our results showed that the amplitude of BW fluctuation was clearly different between batches. It is intuitive to suspect that the number of visits during the day could influence the amplitude of BW fluctuations. For growing-finishing pigs, feeding patterns changed with age from short and frequent meals to long and larger ones (Bigelow and Houpt, 1988; Fàbrega et al., 2003). This was also seen in our data. Pigs in Batch no.5 were observed for $\sim 1$ month only and visited the feeding area with the highest frequency compared with pigs from other batches which were observed over $\sim 2$ months. More frequent visits to the feeding area for pigs in Batch 5 resulted in the lowest fluctuations in daily BW. On the contrary, pigs from Batch 4, with the lowest average number of weight measurements were characterized with the highest variation in BW during a day.

Nielsen et al. (1995) found that the group size in which pigs were kept influenced feeding behavior variables, with pigs kept in larger groups making fewer but longer visits to the feeder compared with smaller groups. In our study, the initial size of the batches was similar. The size of the group 

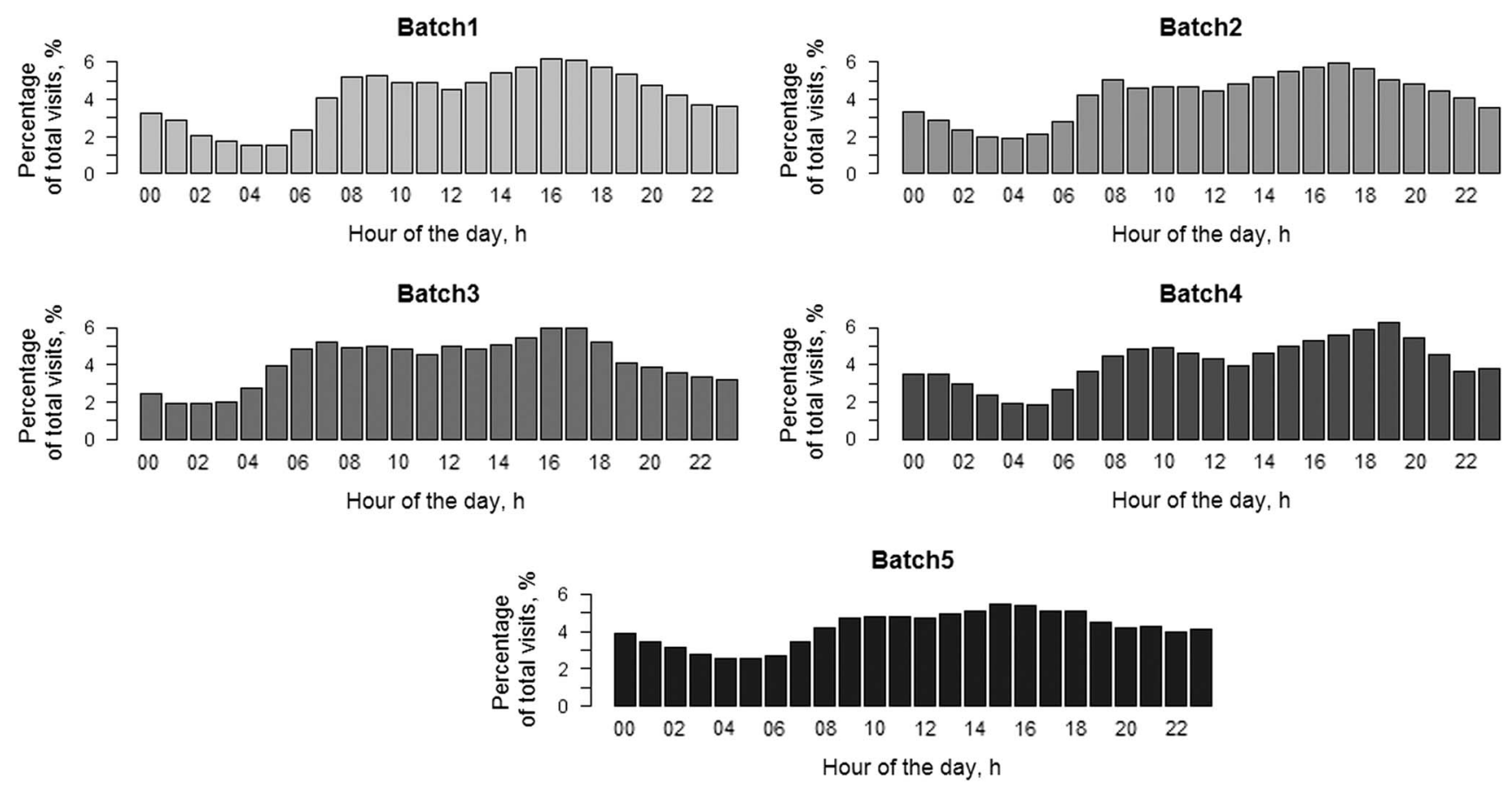

Figure 5 Percentage of total visits to the feeding area (BW measurements) in each batch depending on the hour of the day.

was decreasing with time as the fraction of heaviest pigs was sent to slaughter. As the same slaughter strategy was implemented for all batches, the group size was decreasing at the same pace for all batches. Therefore, differences in the number of visits to the feeding area between batches could mostly be attributed to differences in age.

The main focus of this study was to describe the growth of pigs on an hourly basis. However, future studies on herds using frequent BW monitoring of pigs in large pens could focus on combing behavioral data with BW measurements. Combining this information would allow to study which factors (e.g. the initial BW of inserted pigs, initial variation in BW, etc.) influence the number of visits to the feeding area as well as how the number of visits to the feeding area influences growth rate in a large pen environment.

\section{Observed pattern of visits to the feeding area}

Drinking patterns are closely correlated to feed consumption (Bigelow and Houpt, 1988). Therefore, not surprisingly, diurnal patterns including two stable and distinguished peaks were identified in the drinking behavior of growing pigs in the study by Madsen and Kristensen (2005). Those drinking peaks (morning and afternoon) described by Madsen and Kristensen (2005) coincide with the feeding time and BW peaks found in our study.

Both Hyun et al. (1997) and Young and Lawrence (1994) identified a single feeding peak per day in pigs fed ad libitum at $\sim 0900 \mathrm{~h}$. Other researchers have identified two feeding peaks per day, one in the morning and one in the afternoon (Montgomery et al., 1978; Bigelow and Houpt, 1988; De Haer and Merks, 1992). Differences in the number of feeding peaks have been attributed to the photoperiod that pigs are exposed to (Feddes et al., 1989) and the temperature in the fattening unit (Villagrá et al., 2007).

In our study, pigs had access to daylight and to constant artificial light from a lamp installed above the scale (Figure 1). Pigs in natural conditions are active upon daylight (Curtis, 1937). However, access to artificial light could alter their behavior. As reported by Lay et al. (1999), pigs with constant access to light were more active between 1830 and $0630 \mathrm{~h}$ than pigs subjected to a $12 \mathrm{~h}$ light- $12 \mathrm{~h}$ dark cycle. To our best knowledge, there are no studies that reported pigs' activity in a pen with daylight access and artificial light illuminating the weighing scale in the pen.

In Denmark, the availability of natural light varies according to the season of the year. Since batches were observed during different seasons, different availability of daylight clearly influenced the frequency of visits to the feeding area. For example, pigs from Batch 3 were much more active around $0700 \mathrm{~h}$ compared with pigs from Batch 1 and Batch 2 .

According to Villagrá et al. (2007) if the indoor temperature is close to the thermo neutral zone (between $14^{\circ} \mathrm{C}$ and $24^{\circ} \mathrm{C}$ ), pigs are likely to develop a natural behavior with two peaks of feed consumption and activity during the illuminated period. However, Feddes et al. (1989) reported that changes in temperature on the farm might be sufficient to modify feeding patterns of growing pigs. They found that when the indoor temperature cycles between $26^{\circ} \mathrm{C}$ at $0530 \mathrm{~h}$ and $40^{\circ} \mathrm{C}$ at $1400 \mathrm{~h}$, the diurnal feeding pattern of pigs will be different compared to a scenario with constant temperature $\left(33^{\circ} \mathrm{C}\right)$. They determined that when temperature varied, feed consumption shifted to the coolest part of the thermal cycle. Moreover, the period of highest feed intake occurred earlier in the morning and later at night than in the constant 
temperature treatment. Temperature was not registered in our study therefore the possible influence of temperature on the feeding pattern could not be analysed. Given that the farm had a system regulating indoor temperature, big variations during the production cycle as reported in the above studies were unlikely.

\section{Future application of the model}

Diurnal variation of $\mathrm{BW}$ in pigs can find application in informing the farmer of unexpected growth deterioration for whole batches of pigs or particular individuals. Until recently, obtaining frequent information on BW involved handling animals (moving them to scales located outside of pens) therefore, gaining precise knowledge on BW was difficult and expensive. However, more and more farmers are investing in precision livestock technologies, such as a weight sorting system installed in large pens. In this system, information on BW data is collected on the farm when the pig is visiting the feeding area.

Body weight is a frequently reported trait in biological studies. For example in pig production, we want to know how BW changes with age (Schinckel et al., 2004), during a disease challenge or after applying a therapy (Stygar et al., 2016). However, so far diurnal BW variation has been neglected. According to our results, the difference between morning and evening BW of a pig was significant. Therefore, any frequent BW monitoring tool aiming at detecting, for example, health or herd management problems should account for the diurnal variation of BW in pigs. Moreover, any experiments in which BW is one of the response variables should be conducted so that BW is registered only during a specific part of the day.

The model parameters obtained in this study will be used in a monitoring tool (dynamic linear model (DLM)) based on frequent BW observations. For an example of the application of a DLM to monitoring growth of pigs, a reference to Stygar and Kristensen (2016) is made.

According to the obtained results, parameters describing diurnal variation in BW should be included. Moreover, the obtained parameters (e.g. herd fixed effects, observational and evolution variance as well as autocorrelation) will be used to specify variance components and to set the initial distribution of the parameter vector in the DLM.

The season of the year might also influence the BW of pigs. In Polish conditions, Kościński et al. (2009) found that the highest growth rate in pigs occurs between May and June. They also demonstrated that the weight of 6-month-old pigs plotted by month of birth follows a cosine wave with the highest BW occurring in pigs born in February and the lowest in pigs born in August. Therefore, season is another effect that should be considered for inclusion in the model of pig BW as more data is collected.

\section{Conclusions}

In this paper we described a new model of pig growth applied to frequent BW monitoring. In the proposed model, pig BW was described by the intercept (initial BW), slope (average daily gain during fattening) and a periodic pattern (diurnal fluctuations in BW). In the examined herd, the diurnal amplitude for pig growth ranged from 0.9 to $1.4 \mathrm{~kg}$. Therefore, with modern technologies (such as automatic scaling systems), accounting for daily fluctuations in BW becomes necessary. The proposed mathematical model of growth will be built into a monitoring tool (a DLM with Kalman filter) and applied to farm data in future studies.

\section{Acknowledgments}

This research was supported by the Danish Council for Strategic Research (The PiglT project, Grant number 11-116191). The authors acknowledge The Danish Pig Research Centre and an anonymous farmer for providing data and for valuable information regarding the production unit.

\section{References}

Andretta I, Pomar C, Kipper M, Hauschild L and Rivest J 2016. Feeding behavior of growing-finishing pigs reared under precision feeding strategies. Journal of Animal Science 94, 3042-3050.

Baldwin SP and Kendeigh SC 1938. Variations in the weight of birds. The Auk 55, 416-467.

Bigelow JA and Houpt TR 1988. Feeding and drinking patterns in young pigs. Physiology \& Behavior 43, 99-109.

Clark GA 1979. Body weights of birds: a review. The Condor 81, 193-202.

Cornou C and Kristensen AR 2013. Use of information from monitoring and decision support systems in pig production: collection, applications and expected benefits. Livestock Science 157, 552-567.

Curtis QF 1937. Diurnal variation in the free activity of sheep and pig. Proceedings of the Society for Experimental Biology and Medicine 35, 566-567. De Haer L and Merks J 1992. Patterns of daily food intake in growing pigs. Animal Production 54, 95-104.

Fàbrega $E$, Tibau J, Soler J, Fernández J, Font J, Carrión D, Diestre $A$ and Manteca X 2003. Feeding patterns, growth performance and carcass traits in group-housed growing-finishing pigs: the effect of terminal sire line, halothane genotype and age. Animal Science 77, 11-21.

Feddes J, Young B and DeShazer J 1989. Influence of temperature and light on feeding behaviour of pigs. Applied Animal Behaviour Science 23, 215-222.

Fernández J, Fàbrega E, Soler J, Tibau J, Ruiz JL, Puigvert X and Manteca X 2011. Feeding strategy in group-housed growing pigs of four different breeds. Applied Animal Behaviour Science 134, 109-120.

Grolemund $G$ and Wickham $H$ 2011. Dates and times made easy with lubridate. Journal of Statistical Software 40, 1-25.

Hoy S, Schamun S and Weirich C 2012. Investigations on feed intake and social behaviour of fattening pigs fed at an electronic feeding station. Applied Animal Behaviour Science 139, 58-64.

Hyun Y, Ellis M, McKeith F and Wilson E 1997. Feed intake pattern of group-housed growing-finishing pigs monitored using a computerized feed intake recording system. Journal of Animal Science 75, 1443-1451.

Kościński K, Kozłowska-Rajewicz A, Górecki M, Kamyczek M and Różycki M 2009. Month-of-birth effect on further body size in a pig model. HOMO-Journal of Comparative Human Biology 60, 159-183.

Kristensen AR and Jensen DB 2015. The intelligent pig barn. In Proceedings of the: International Conference on Pig Welfare: Improving Pig Welfare-What Are the Ways Forward, 29-30 April 2015, Copenhage, Denmark, pp. 34-37.

Krogsdahl J 2015. May PiglT Report No. 7. Description of Herd with Large common pens. Retrieved on 25 February 2015 from http://pigit.ku.dk/ publications/PiglT-Report7.pdf

Lay D, Buchanan H and Haussmann M 1999. A note on simulating theobserver effect' using constant photoperiod on nursery pigs. Applied Animal Behaviour Science 63, 301-309.

Macleod R, Barnett P, Clark J and Cresswell W 2005. Body mass change strategies in blackbirds Turdus merula: the starvation-predation risk trade-off. Journal of Animal Ecology 74, 292-302. 


\section{Stygar, Dolecheck and Kristensen}

Madsen TN and Kristensen AR 2005. A model for monitoring the condition of young pigs by their drinking behaviour. Computers and Electronics in Agriculture 48, 138-154.

Mäntysaari P and Mäntysaari E 2015. Modeling of daily body weights and body weight changes of Nordic Red cows. Journal of Dairy Science 98, 6992-7002.

Montgomery G, Flux D and Carr J 1978. Feeding patterns in pigs: the effects of amino acid deficiency. Physiology \& Behavior 20, 693-698.

Nielsen BL, Lawrence $A B$ and Whittemore CT 1995. Effect of group size on feeding behaviour, social behaviour, and performance of growing pigs using single-space feeders. Livestock Production Science 44, 73-85.

Niemi J, Sevón-Aimonen M-L, Stygar A and Partanen K 2015. The economic and environmental value of genetic improvements in fattening pigs: an integrated dynamic model approach. Journal of Animal Science 93, 4161-4171.

Pinheiro J, Bates D, DebRoy S and Sarkar D 2017. R Core Team (2017) nlme: linear and nonlinear mixed effects models. R package version 3.1-126. Retrieved on 15 February 2017 from http://CRAN.R-project.org/package=nlme

Pinheiro JC and Bates DM 2000. Mixed-effects models in S and S-Plus. SpringerVerlag, New York.
R Core Team 2014. R: A language and environment for statistical computing. R Foundation for Statistical Computing, Vienna, Austria. http://www.R-project.org/ Schinckel AP, Ferrel J, Einstein ME, Pearce SM and Boyd RD 2004. Analysis of pig growth from birth to sixty days of age1. The Professional Animal Scientist 20, 79-86.

Stygar AH and Kristensen AR 2016. Monitoring growth in finishers by weighing selected groups of pigs - a dynamic approach. Journal of Animal Science 94, 1255-1266.

Stygar AH, Niemi JK, Oliviero C, Laurila T and Heinonen M 2016. Economic value of mitigating Actinobacillus pleuropneumoniae infections in pig fattening herds. Agricultural Systems 144, 113-121.

Toft N, Kristensen AR and Jørgensen E 2005. A framework for decision support related to infectious diseases in slaughter pig fattening units. Agricultural Systems 85, 120-137.

Villagrá A, Althaus RL, Lainez M, Martinez AB and Torres AG 2007. Modelling of daily rhythms of behavioural patterns in growing pigs on two commercial farms. Biological Rhythm Research 38, 347-354.

Young $R$ and Lawrence A 1994. Feeding behaviour of pigs in groups monitored by a computerized feeding system. Animal Science 58, 145-152. 